\title{
La 15e Conférence internationale Tournesol (Toulouse, 13-15 juin 2000)
}

\section{5th International sunflower conference The 15th International Sunflower Conference (13-15 June 2000, Toulouse)}

Oléagineux, Corps Gras, Lipides. Volume 7, Numéro 6, 465-6, Novembre - Décembre 2000, La filière

Auteur(s) : Bernard SchweiSguth, Inra-UR GAP, Centre de Dijon, 21110 Bretenières.

Résumé : L'ambition de la Conférence* était de faire un état des connaissances et des travaux de recherche concernant le tournesol dans des domaines très différents : économie, technologie et transformation industrielle, agronomie et environnement, biotechnologie, génétique et sélection. Des synthèses ont été faites par des personnalités invitées, lors de séances plénières, accessibles à tous, spécialistes ou non des domaines traités. Par ailleurs, les résultats et les programmes de recherches de tous les pays producteurs de tournesol étaient présentés par des affiches - environ 300, regroupées en 15 sections. Des ateliers ont été organisés pour faire des synthèses, donner l'occasion aux chercheurs de faire de très courtes présentations et de confronter leurs travaux et résultats. Ces ateliers d'une durée de $1 \mathrm{~h} 30$ avaient lieu dans des salles séparées et non autour des affiches exposées. Ils ont connu un grand succès.

Summary : The aim of the Conference was to give updates on all aspects of the sunflower research, from economics to biotechnologies and to allow researchers from public institutions and private companies to present their work and discuss their results. The four plenary sessions were devoted to : Economics, Technology, Agronomy and Environment and Genetics, Breeding and Biotechnology. Three hundred posters were shown in 15 sections and were the matter for exchanges and discussions in 17 workshops. The main topics of these workshops were technology and industrial transformation, new uses for oil, crop physiology and crop management with an emphasis on water stress and irrigation, grain quality and oil composition, crop protection and disease resistance, breeding for yield and genetic $x$ environnement interaction, biomolecular genetics, biotechnology and genetic resources.

\section{ARTICLE}

De l'ensemble des présentations - séances plénières et ateliers - on peut tirer quelques points forts dans les différents domaines.

Économie. L'huile de tournesol est une des huiles majeures issues de graines (la $3^{\mathrm{e}}$ au niveau mondial). Elle est soumise à une très forte compétition (exposé de $\mathrm{H}$. Rieux, de Cereol). Selon les pays et les régions, la production du tournesol est plus ou moins compétitive vis-à-vis d'autres cultures (céréales, colza) : bien placée en Argentine où elle est une production agricole majeure ( $1^{\text {er }}$ producteur mondial), elle est, en revanche, peu compétitive en France (rendements assez faibles et peu stables) (exposé de A. Pouzet, Cetiom). 
Technologie, transformation industrielle, nouveaux usages. II a beaucoup été question de tournesol à haute teneur en acide oléique, en particulier du fait de son intérêt en nutrition humaine (exposé de Bernadette Delplanque, Inserm, voir OCL (2000) 7 : 243-55).

Par ailleurs, dans le domaine non alimentaire, le tournesol est la source de beaucoup de produits valorisables à partir des graines, des capitules, des tiges... (exposé de Élisabeth Borredon, Ensat). Dans l'atelier consacré à ces sujets, on a beaucoup discuté d'oléochimie (travaux français notamment).

Biologie moléculaire. Comparé à une espèce comme le maïs, le tournesol fait figure de parent pauvre en ce qui concerne les applications de la biologie moléculaire. Pourtant Steve Knapp (Université de Corvallis, Oregon, États-Unis) a pu faire un panorama impressionnant de ce qui a déjà été fait en Amérique comme en Europe dans le domaine de la cartographie génétique et du marquage de caractères d'intérêt. II a aussi insisté sur les possibilités offertes par les techniques développées, en particulier par son laboratoire. Il a plaidé pour une mise en commun de ces ressources.

Les exposés sur les variations de la composition en acides gras des huiles (A. Bervillé, Inra, Montpellier), sur les gènes de résistance aux maladies ( $\mathrm{S}$. Mouzeyar, Université de Clermont-FerrandII), sur la phylogénie des Helianthus (Ed. Schilling, Université de Knoxville, Tennessee, États-Unis) ont montré le rôle clé joué par les méthodes et les techniques de la biologie moléculaire dans ces domaines d'investigation. On retrouve ce rôle de la biologie moléculaire dans de nombreux travaux rapportés lors des ateliers posters.

Biotechnologie. Les spécialistes de la biologie cellulaire disent que rien de bien nouveau n'a été annoncé. Mais différentes techniques de transformation génétique ont pu être confrontées. Quelques exemples de transformation génétique réussie ont été donnés : résistance aux maladies et aux parasites.

Écophysiologie. Antonio Hall (Université de Buenos Aires) a fait une remarquable revue des problèmes non résolus en écophysiologie : par exemple, le rôle de la photopériode dans le développement de la plante (il n'y a pas de bon modèle du déterminisme de la précocité de floraison), les effets combinés de la densité de peuplement, du rayonnement, du génotype sur le remplissage du grain et la teneur en huile, le rôle du caractère stay green... Ces thèmes ont été repris lors des ateliers posters. Les Argentins sont très présents dans ce vaste domaine de recherche.

L'ambition des écophysiologistes est de proposer des modèles de " fonctionnement " de la culture utiles dans différents domaines : sélection (définition d'idéotypes : structure de la plante, phénologie...), conduite des cultures (simulation, outils d'aide à la décision : par exemple l'irrigation).

Conduite des cultures. La culture du tournesol est réputée peu coûteuse en intrants, particulièrement en azote et en eau. Néanmoins, une conduite économe, efficace des points de vue agronomique et économique, nécessite beaucoup d'attention. Les travaux argentins, italiens, marocains et français se sont attachés à définir les meilleures conduites dans des conditions variées et plus spécifiquement les conditions sèches. Ils attendent beaucoup des modèles de croissance et de développement qui permettront de construire des outils d'aide à la décision. 
Sélection pour le rendement. La séance plénière " économie » a clairement montré que, dans un pays comme la France, la productivité et sa stabilité sont trop basses pour que la culture soit bien compétitive. Les premiers essais de type " progrès génétique " argentins montrent le saut de productivité dû aux hybrides, mais n'apportent que peu d'indication sur les caractères à sélectionner pour faire de nouveaux progrès. Les études d'aptitude à la combinaison entre origines génétiques pour structurer la variabilité sont encore trop peu développées pour permettre de conduire la sélection pour le rendement de façon plus rationnelle. II y a beaucoup à faire pour mieux comprendre les bases physiologiques de la production de graine et d'huile et pour mieux connaître et exploiter la variabilité génétique de l'espèce.

Protection phytosanitaire, résistance aux maladies et aux parasites ont été des sujets très présents, avec les équipes américaines et surtout françaises qui sont très en pointe sur ces sujets. On doit signaler la conception globale de lutte contre le mildiou, présentée par Denis Tourvieille (Inra), utilisant à la fois les fongicides et les résistances génétiques, qui est un modèle du genre. Sur ce même couple hôte/parasite, la compréhension des mécanismes de résistance a bien avancé grâce aux outils de la biologie moléculaire (exposé de S. Mouzeyar, UMR, Inra, Université de Clermont-II).

Les interventions dans l'atelier " résistance verticale » furent surtout françaises, sur la résistance au mildiou. Dans l'atelier " résistance horizontale ", la résistance au Sclerotinia a été le thème prépondérant (60\% des communications) avec des interventions d'Argentins, d'Américains, de Roumains, d'Italiens et de Français. Une nouveauté intéressante est la transformation du tournesol par un gène d'oxalate oxydase du blé. Le niveau de résistance est amélioré mais, avec la lignée utilisée, il atteint seulement celui des témoins résistants classiques.

Le phoma du tournesol est une maladie qui « monte ", du moins en France, et qui préoccupe de plus en plus les pathologistes.

Un premier exemple de lutte contre les insectes ravageurs par un gène Bt introduit par génie génétique a été présenté par une équipe américaine.

Depuis l'avènement des variétés hybrides, c'est certainement dans le domaine des résistances aux maladies que la sélection a fait les progrès les plus importants.

En guise de conclusion, il est intéressant de revenir sur les variétés de tournesol à haute teneur en acide oléique. La première variété de ce type, Pervenets, a été obtenue par mutagenèse en 1976 par le Dr Soldatov qui était présent à la conférence. À partir de celle-là la sélection classique a créé d'autres variétés mieux adaptées aux diverses conditions de culture. Les industriels s'y sont intéressés pour l'alimentation humaine et la lipochimie. Leur culture s'est étendue. Nous avons eu lors de la Conférence des témoignages sur les études conduites dans ces différents domaines. Enfin, une équipe Inra (A. Bervillé à Montpellier) a analysé la biosynthèse de ces huiles et décrit les gènes impliqués (delta12 désaturase). Nous savons que, pour être cultivées sans restriction, ces variétés doivent encore être améliorées pour leur résistance aux maladies. En quelques jours nous avons eu un panorama concernant 25 ans de recherche sur ces nouvelles variétés, de leur obtention à leur utilisation, et de nouvelles perspectives pour leur amélioration. 
C'est une bonne illustration de l'objectif de cette conférence : faire se rencontrer les chercheurs de disciplines scientifiques, domaines d'application et générations différents, tous impliqués dans le tournesol, pour échanger, confronter et resituer leurs propres travaux dans un ensemble plus vaste.

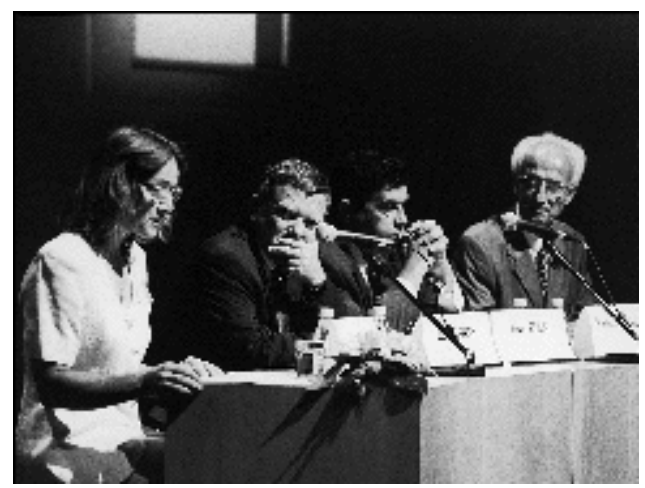

Photo. $15^{e}$ Conférence internationale

Tournesol à Toulouse. De gauche à droite : Felicity Vear, présidente de I'ISA et présidente du comité d'organisation, Jean Glavany, ministre français de I'Agriculture, Xavier Beulin, président de la FOP et Bertrand Schweisguth, président du comité scientifique de la Conférence (photo Lot). 